\title{
Greeting Messages from President of Japanese Society of Hypertension (JSH) The challenge of JSH: moonshot for "Conquest of hypertension in Japan"
}

\author{
Hiroshi Itoh ${ }^{1}$ \\ Received: 15 May 2019 / Accepted: 15 May 2019 / Published online: 5 July 2019 \\ (c) The Japanese Society of Hypertension 2019
}

From September 2018, I have been appointed President of Japanese Society of Hypertension. It is an extreme honor, and I intend to dedicate myself for further development of JSH, established through tireless efforts of the leading doctors in the field of hypertension in Japan. Continued and valuable guidance and encouragement from the Society members would be greatly appreciated.

My overarching vision for the Society is: "Conquest of hypertension in Japan" and "Establishment of Society's global presence." Mission of a clinical society is the elimination of target diseases. As a single disease, "Hypertension" is a most serious contributing factor to death worldwide after smoking. In 2008, it is estimated that more than 1 billion people over the age of 25 worldwide, or 1 in 3, are diagnosed with hypertension. Also in Japan, the number of patients with hypertension has reached 43 million, and the medical expenses spent for hypertension is well over 1 trillion yen. However, the number of Japanese hypertension patients with the correct treatment accounts for less than $30 \%$. We have come to the time for all of our Society members to seriously reason the fact.

The Japanese Society of Hypertension, established in April 1978 with approximately 300 members, has grown through the wisdom, creativity, and effort of many doctors into an organization of 5,157 members (4,312 regular members and 845 associate members as of November 20 , 2018), consisting of 719 hypertension specialists, 324 hypertension/cardiovascular disease prevention and treatment instructors, 268 Fellow of Japanese Society of Hypertension (FJSH), and 325 councilors. During the period, the basic research on hypertension and its complications in Japan led the world's hypertension research, including the

Hiroshi Itoh

hiito@keio.jp

1 Department of Endocrinology, Metabolism and Nephrology Keio University School of Medicine, Tokyo, Japan discovery of blood pressure control hormones, and the development of drugs based on the results has been conducted. In 2000, the Japanese Society of Hypertension Guidelines for the Treatment of Hypertension 2000 was published. Since 2004, the guidelines have been updated every 5 years, and in 2019, the guidelines is announced based on systematic review supported by high-quality evidence. Our guidelines are one of the most commonly read guidelines in Japan and serve as an example of other guidelines. On the other hand, clinical research issues occurred in the past, however, under the leadership of former president, Dr. Satoshi Umemura and Dr. Sadayoshi Ito, the Society members worked together with a serious attitude to review the public selection system of directors, strengthen the audit system, and other visualization of Society operations, aimed at the promotion of proper clinical research such as establishment of ethical standards. Furthermore, in order to improve the awareness of and the skills to cope with hypertension among professionals, such as public health nurses, nurses, pharmacists, registered dietitians, and clinical laboratory technicians, a certification system for the "Hypertension and Cardiovascular Disease Prevention and Treatment Instructor" was established and disseminated in cooperation with the Japanese Association of Hypertension. In addition, low-salt activities for citizens have been steadily achieved through efforts of many professionals.

As described above, the bona fide activities of Society accumulated over 40 years have certainly reaped some results. Such activities are a true approach to control hypertension and we should continue to strengthen and expand them further. However, there is still a reality that hypertension cannot be eradicated. The fact tells us that the conquest of hypertension requires a fundamental paradigm shift in hypertensiology. We also need an approach from a completely new angle that we have not challenged. The challenge to "hypertensiology in a different dimension" is sought. And I think that it is quite possible based on the robust foundation of our Society cultivated so far. 
With rising crisis consciousness, the JSH Future Plan was established in 2018. The declaration was made as our Society's principle, "We shall research and practice the best medical care for hypertension and carry out activities to realize healthy and bright society for the people of Japan" with a slogan "Good Blood Pressure for Lively 100 Years," and a pledge was made to the public to "Reduce the number of people with hypertension by 7 million in 10 years and extend their healthy life expectancy" as a concrete goal.

Three pillars to achieve the goal were set:

(1) Medical care system: Construction of lifelong hypertension treatment system (Life-time care),

(2) Academic research: Promotion of hypertension research and realization of "JSH Future Plan", and

(3) Social enlightenment: Development of society where citizens manage their blood pressure at own initiative.

In order to establish "Hypertension in a different dimension," the concept of hypertension needs to be changed first. Hypertension is not just a condition with high blood pressure, but central pathophysiology of "metabolic domino" which I presented in 2003. Metabolic disorders, such as obesity, impaired glucose tolerance, and lipid abnormalities, that coincide with hypertension due to impairment of homeostasis produced in the deflection of lifestyle such as diet, exercise, sleep, and social behavior, are included in a pathological states under "Hypertension in a broad sense, and not as "Comorbidity of hypertension." A series of diseases including chronic kidney disease (CKD), cerebrovascular disorder, heart failure, cognitive disorder, and cancer are now called "Non-Communicable Diseases; NCDs," which are positioned as the most important disease entity by WHO. Hypertension should be deemed as a collective of NCDs. Therefore, our Society members should be familiar with all of the realities of NCDs.

Based on the above, the conquest of hypertension requires both (i) realization of Precision Medicine for hypertension that ensures thoroughly individualized medical care capitalized on various omics-based analysis techniques including genome analysis, IT, device, and robotic technologies, and $\mathrm{AI} / \mathrm{big}$ data processing technologies, and (ii) action on hypertension based on population as a while to reduce the risks of chronic disease in a long-term span from prevention.

(i) Precision Medicine for hypertension: Genomic science, chronological acquisition and processing of big data across the general population, and utilization of new devices are the most advanced research area (Data Science). In hypertensiology, it is hoped that young people, who are familiar with the area and spirited to create a model case, will be assigned to
Data Science under the leadership of the Society. We believe that our activity approach aimed at "Digital Hypertension" would also attract young doctors who have not been mobilized so far, and lead to their participation in the Society. It is desirable to accurately predict the future of each and every patient with hypertension and those at risk and perform early intervention for realization of Pre-emptive medicine.

(ii) Population Health Management of hypertension: Needless to say that it is desirable to strengthen the dynamic integration of clinical fields, including not only university hospitals but also core community hospitals, practitioners, public health nurses, nurses, pharmacists, registered dietitians, and clinical laboratory technicians. But that is not enough. Some statistical results show that healthcare contributes only $10-20 \%$ of health realization. If we truly seek conquest of hypertension, the intervention would require comprehensive understanding of extremely broad areas, from policy recommendations, assessment of the influence of legislations, understanding of the behavior principle of individuals in society and their collective trends (including understanding of economic status and genetic background).

In order to conquer hypertension, hypertensiology needs to be tackled as Convergence Science, a scientific approach in which knowledge of all fields is integrated on the same platform, leading to health outcomes. For this reason, it is desirable to try and invite and mobilize not only healthcare professionals in the medical and healthcare fields but also those in broader and diverse fields to participate in the Society.

For the realization of JSH Future Plan, I set up three specific action plans that we will work intensively and formed the taskforces.

\section{Medical Care System}

Proactive promotion of measures against hypertension and its complications (heart failure, etc.) and strengthening of cooperation with other academic societies

\section{Academic Research}

A new approach to detect dysregulation of blood pressure" in the AI era and development of hypertension big data science

\section{Social Enlightenment}

Construction of a model town with controlled "hypertension" ("Hypertension Zero Town”)

In 2022, the International Society of Hypertension (ISH) KYOTO 2022 is scheduled to be held in Kyoto, for which I have been appointed Chairman. I see the timing as an interim presentation of the outcomes of Future Plan looking 
forward to the next 10 years. I have a dream that the ISH KYOTO 2022 would become a venue to have participants from around the world understand the real capabilities of Japanese hypertensiology, and proudly show our approach to the world as Japan standard know-how for the conquering of hypertension.

In September 2018, at the 41st Annual Meeting of the Japanese Society of Hypertension (chaired by Dr. Naoyuki Hasebe) held in Asahikawa immediately after the earthquake, we pledged the "Asahikawa Oath" for the launch of a new system of the Society. In other words, in the spirit that all Society members are peers, a vision for the Society members was upheld after the word, "PEER."

I would like to share this spirit with all members of the Society.

\section{PEER}

P: Participation

For love of the Society, all members shall participate.

E: Engagement

Members shall find what can be done to improve the Society and execute with a sense of responsibility.

E: Encouragement
Members shall respect and encourage activities of other peers.

R: Relay

Members shall pass on to the next generation what was achieved and what could not be achieved.

I would like to express my gratitude to the Society members for their understanding on my thoughts, approaches to activities, and frameworks for the Society. I sincerely hope that we will work together towards the realization of better Society and construction of "Affluent and bright society with conquered hypertension."

Finally, I would like to end this message with my favorite words of the 16th US President, Abraham Lincoln.

"The best way to predict the future is to create it."

\section{Compliance with ethical standards}

Conflict of interest The author declares that he has no conflict of interest.

Publisher's note: Springer Nature remains neutral with regard to jurisdictional claims in published maps and institutional affiliations. 